\title{
Fibrinogen and the prediction of residual obstruction manifested after pulmonary embolism treatment
}

\author{
Benjamin Planquette ${ }^{1,2,3}$, Olivier Sanchez ${ }^{1,2,3}$, James J. Marsh ${ }^{4}$, \\ Peter G. Chiles ${ }^{4}$, Joseph Emmerich ${ }^{1,5}$, Grégoire Le Gal ${ }^{6}$, Guy Meyer ${ }^{1,2,7,8}$ \\ Tanya Wolfson", Anthony C. Gamst ${ }^{9}$, Roger E. Moore ${ }^{10}$, Gabriel B. Gugiu ${ }^{10}$ and \\ Timothy A. Morris ${ }^{4}$
}

Affiliations: ${ }^{1}$ Université Paris Descartes, Sorbonne Paris Cité, France. ${ }^{2}$ Service de Pneumologie et Soins Intensifs, Hôpital Européen Georges Pompidou, AP-HP, Paris, France. ${ }^{3}$ INSERM UMR-S 1140, Paris, France. ${ }^{4}$ Dept of Medicine, Division of Pulmonary and Critical Care Medicine, University of California, San Diego, CA, USA. ${ }^{5}$ Médecine Vasculaire - Cardiologie, Centre de Diagnostic et de Thérapeutique, Hôpital Hôtel Dieu, AP-HP, Paris, France. ${ }^{6}$ Dept of Medicine, Ottawa Hospital Research Institute, University of Ottawa, Ottawa, ON, Canada. ${ }^{7}$ INSERM CIC-1418, Paris, France. ${ }^{8}$ INSERM UMR-S 970, Paris, France. ${ }^{9}$ Computational and Applied Statistics Laboratory (CASL), San Diego Supercomputer Center, University of California San Diego, San Diego, CA, USA. ${ }^{10}$ Beckman Research Institute of the City of Hope, Duarte, CA, USA.

Correspondence: Timothy A. Morris, Division of Pulmonary, Critical Care and Sleep Medicine, University of California, San Diego Healthcare, 200 West Arbor Drive, San Diego, CA 92103-8378, USA. E-mail: t1morrisवucsd.edu

@ERSpublications

Fibrinogen helps predict residual pulmonary vascular obstruction after pulmonary embolism: results from the PROMPT study http://ow.ly/G7pY30m2HU6

Cite this article as: Planquette B, Sanchez O, Marsh JJ, et al. Fibrinogen and the prediction of residual obstruction manifested after pulmonary embolism treatment. Eur Respir J 2018; 52: 1801467 [https://doi. org/10.1183/13993003.01467-2018].

ABSTRACT Residual pulmonary vascular obstruction (RPVO) and chronic thromboembolic pulmonary hypertension (CTEPH) are both long-term complications of acute pulmonary embolism, but it is unknown whether RPVO can be predicted by variants of fibrinogen associated with CTEPH.

We used the Akaike information criterion to select the best predictive models for RPVO in two prospectively followed cohorts of acute pulmonary embolism patients, using as candidate variables the extent of the initial obstruction, clinical characteristics and fibrinogen-related data. We measured the selected models' goodness of fit by analysis of deviance and compared models using the Chi-squared test.

RPVO occurred in 29 (28.4\%) out of 102 subjects in the first cohort and 46 (25.3\%) out of 182 subjects in the second. The best-fit predictive model derived in the first cohort $(p=0.0002)$ and validated in the second cohort $(p=0.0005)$ implicated fibrinogen $B \beta$-chain monosialylation in the development of RPVO. When the derivation procedure excluded clinical characteristics, fibrinogen B $\beta$-chain monosialylation remained a predictor of RPVO in the best-fit predictive model $(p=0.00003)$. Excluding fibrinogen characteristics worsened the predictive model $(\mathrm{p}=0.03)$.

Fibrinogen B $\beta$-chain monosialylation, a common structural attribute of fibrin, helped predict RPVO after acute pulmonary embolism. Fibrin structure may contribute to the risk of developing RPVO. 


\section{Introduction}

After acute pulmonary embolism, thrombotic material in the pulmonary arteries typically disintegrates, although in some cases it transforms into permanent obstructing scars [1]. Residual pulmonary vascular obstruction (RPVO) of $\geqslant 10 \%$ is common after pulmonary embolism and can diminish physical activity, reduce quality of life and worsen overall prognosis [2-7]. RPVO is associated with dyspnoea [8], hypoxaemia [9], gas exchange deficits [9], exercise intolerance [2] and other serious clinical impairments [9-11]. RPVO incurs the risk of recurrence [12] and of chronic thromboembolic pulmonary hypertension $(\mathrm{CTEPH})[2,13]$, an uncommon $[14,15]$ but severe complication of pulmonary embolism [16, 17] in which obstruction is extensive and pulmonary arterial pressure rises [18].

The mechanisms by which fibrin-rich thromboemboli transform into permanent obstructions characteristic of RPVO and of CTEPH are incompletely understood. RPVO is not associated with "thrombophilia" or with impaired fibrinolytic enzymes [2]. We observed that fibrin itself derived from some patients with CTEPH forms clots resistant to lysis [19, 20] with aberrations in molecular configuration [21] and clot structure [22] that reflect otherwise rare fibrinogen gene mutations [20]. CTEPH-associated dysfibrinogenemia may be a model for more common fibrin characteristics that might tip the balance from clot resolution towards RPVO.

To gain insights into the development of RPVO, the Prediction of Residual Obstruction Manifested after Pulmonary Embolism Treatment (PROMPT) study followed two cohorts of patients after acute pulmonary embolism to determine the best predictors of RPVO. We investigated whether fibrinogen post-translational modifications and other common fibrin structural attributes might help predict the development of RPVO after acute pulmonary embolism. If fibrin properties are consistently associated with RPVO, then they might be implicated as a mechanism of its development.

\section{Methods}

\section{Study design and study population}

We used clinical data and biological material from two single-centre, prospective, observational cohort studies conducted on separate groups of acute pulmonary embolism patients in an academic teaching hospital (Hôpital Européen Georges Pompidou, Paris, France). The two studies were approved by the local ethics committee. All patients provided written informed consent before enrolment.

For cohort 1, we enrolled consecutive patients with pulmonary embolism from our centre who had been included in the FARIVE study, a multicentre case-control study that evaluated the interactions of environmental, genetic and biological risk factors on the risk of a first venous thromboembolism (VTE). The study included consecutive subjects who were aged $>18$ years and had their first symptomatic occurrence of pulmonary embolism. Patients were excluded if they had active cancer, unless they had been treated successfully and had no evidence of recurrence in the previous 5 years. In addition, patients were excluded if they had short life expectancies secondary to other associated pathologies, anticipated impossibility of follow-up or a previously diagnosed thrombophilia.

For cohort 2, we enrolled consecutive patients with pulmonary embolism followed-up at our centre between February 1999 and May 2006 who had not been enrolled in the FARIVE study [2]. This study was designed to assess the clinical significance of residual pulmonary vascular obstruction after an episode of acute pulmonary embolism. Patients were included if they were aged $>18$ years and had completed $\geqslant 3$ months of oral anticoagulant therapy without having recurrent pulmonary embolism.

In both cohorts, the diagnosis of pulmonary embolism was established through high-probability lung scintigraphy, thoracic computed tomography (CT) scan, pulmonary angiography, sonography showing the existence of a proximal venous thrombosis in the absence of a differential diagnosis for the reported pulmonary symptoms or nondiagnostic pulmonary scintigraphy associated with lower extremity ultrasound disclosing proximal deep vein thrombosis. The type and duration of long-term therapy was left to the discretion of physician in charge of the patients. Standard practice was to treat provoked pulmonary embolism for $\geqslant 3$ months and unprovoked pulmonary embolism for $\geqslant 6$ months.

\section{Residual pulmonary vascular obstruction}

All patients underwent a standardised ventilation/perfusion ratio $\left(V^{\prime} / Q^{\prime}\right)$ lung scan $\geqslant 6$ months after the acute pulmonary embolism [23]. A trained investigator (BP) blinded to all other study data identified and scored mismatched perfusion defects according to a prespecified protocol [24]. We defined RPVO as a residual vascular obstruction of $\geqslant 10 \%$, which corresponds to an amputation of at least two pulmonary segments (the minimal obstruction defining a high-probability result on diagnostic $V^{\prime} / Q^{\prime}$ lung scans [25]) and is associated with dyspnoea and exercise intolerance after acute pulmonary embolism [2]. 


\section{Demographic and clinical data}

A standardised tool was used upon study enrolment to record clinical data (including the type of acute therapy and the duration of long-term therapy) and conduct a structured interview. Except for the fibrinogen-related laboratory data described in the following section, all laboratory values were measured in the clinical laboratory of Hôpital Européen Georges Pompidou. Physicians blinded to the other data of each patient used a validated score to calculate the initial pulmonary vascular obstruction on the original diagnostic CT scan [26] or $V^{\prime} / Q^{\prime}$ scan [24]. None of the subjects had pulmonary angiography as the initial diagnostic test.

\section{Fibrin structural attributes}

Upon enrolment, blood samples were collected by venipuncture into anticoagulant-containing tubes. Plasma was separated by two consecutive centrifugations and stored at $-70^{\circ} \mathrm{C}$ for analysis. Plasma fibrinogen levels were measured in a clinical laboratory at the time of blood collection.

Research assays were performed on fibrinogen that had been purified from citrated plasma by ethanol precipitation and exposure to Gelatin Sepharose (GE Healthcare, Pittsburg, PA, USA) to remove residual fibronectin, followed by dilution to $4 \mathrm{mg} \cdot \mathrm{mL}^{-1}$ [20]. Online supplementary appendix 1 includes the detailed assay methodologies, which have been reported previously [19, 20, 22]. They are described briefly below.

Fibrinogen, thrombin and calcium chloride were combined to form fibrin clots in the amounts appropriate for each structural assay. We determined the turbidity of the fibrin clots, which corresponds to fibrin network fibril dispersal and branching as validated by microscopy [20, 22]. We measured fibrin clot susceptibility to lysis through the rates of turbidity decrease, measured consecutively after clots were exposed to a mixture of plasminogen and tissue plasminogen activator [20]. We measured fibrin clot permeability, which reflects the organisation with which fibrin polymers are formed [22], by the rate of passage of permeability buffer through fibrin clots. More organised fibrin networks with large pores have higher flow compared to disorganised fibrin networks with small pores. We measured the degree that fibrin crosslinks its $\alpha$-chains using sodium dodecyl sulfate polyacrylamide gel electrophoresis as a reflection of the potential to form stabilised clots during thrombosis [19]. We used $\mathrm{I}^{125}$-labelled antibodies specific for the $\beta_{15-42}$ peptide sequence chain (anti- $\beta_{15-42}$ ) to measure the accessibility within the fibrin clots of the fibrin $\beta$-chain amino termini (residues 15-42), which are implicated in a variety of physiological events that affect thrombus remodelling into intravascular scars [27-29].

We measured precise fibrinogen masses using liquid chromatography, followed by mass spectrometry (LC/ MS) to identify post-translational modifications [20]. We analysed the LC/MS spectra representing the fibrinogen $A \alpha$-chains, $B \beta$-chains and $\gamma$-chains and measured the proportional heights of mass peaks corresponding to variants we had observed in CTEPH-associated dysfibrinogenaemias and any other mass peaks that represented $\geqslant 10 \%$ of the associated fibrinogen chains.

\section{Statistical analysis}

Categorical variables are presented as $\mathrm{n}(\%)$ and continuous variables are presented as mean \pm SD. All available demographic, clinical and fibrinogen data were compared between the two cohorts, using t-tests, Wilcoxon-Mann-Whitney tests, Fisher's exact tests or Chi-squared tests, as appropriate. Univariate assessment of predictors of RPVO was performed for each cohort, using the same tests, as appropriate.

Because the interaction of lysis, the inflammation and cellular remodelling that leads to RPVO probably develops through a complex interaction between clinical factors and biological factors, the careful selection of a predictive model was critical to our goal. A joint predictive effect would be obscured if simplistic univariate analyses were used [30]. For this reason, we used a stepwise multivariate logistic regression procedure based on the Akaike information criterion (AIC) $[30,31]$ to select the most parsimonious and accurate multivariable prediction model for RPVO in each cohort, using as candidate predictors both clinical risk factors and fibrin structural properties. If the best predictive models selected in this fashion depended on fibrin structural attributes, then the analysis would support the role of fibrin properties in the development of RPVO after acute pulmonary embolism. The AIC-based approach avoids selecting models solely on the basis of apparent significance of the individual terms or global fit, both of which are likely to be overstated; overfitting is penalised [31]. We used deviance-based goodness of fit, a standard logistic regression metric, to assess the quality of the selected models in each cohort.

Multivariable modelling was performed on information from subjects who had complete data (without missing values), to enable model comparisons. For cohort 1, AIC-based stepwise logistic regression was used to identify the best clinical and demographic predictors of RPVO. Then, AIC-based stepwise logistic regression was used to select the best model to predict RPVO, using as candidate predictors each patient's 
previously identified relevant demographic and clinical measures, as well as the results of the fibrinogen experiments we described. We used the receiver operating characteristic (ROC) curve [32] to find the best probability cut-off for predicting RPVO and assessed the model's sensitivity and specificity. We then validated the model derived in cohort 1 by using it to predict RPVO in cohort 2 .

To confirm the role of specific fibrin structural attributes in RPVO, we performed the prediction model selection process again in cohort 1 and included in the candidate predictor pool only the fibrinogen properties and the initial degree of obstruction (excluding the clinical data). The initial degree of obstruction is such a strong predictor of residual obstruction [2] that all predictive models would include it. We were interested in finding additional, independently predictive measures. Finally, to further verify the role of fibrinogen, we used a Chi-squared test of residual deviances to determine whether the model that included the initial obstruction as well as fibrinogen properties were significantly better predictors of RPVO than the model that excluded fibrinogen properties.

To confirm the generalisability of individual RPVO predictors identified in cohort 1, we repeated the multivariate modelling process in cohort 2, but limited the candidate variables to those that were disclosed by the cohort 1 analysis. We reasoned that variables predictive of RPVO in both cohorts might be implicated in RPVO development in the general population of patients with acute pulmonary embolism. If a variable derived from cohort 1 was not included in the optimal prediction equation generated for cohort 2 , or if its coefficient was in the opposite direction, we reasoned that it would be less likely to be predictive of RPVO in other pulmonary embolism populations.

\section{Results}

\section{Subject populations}

From January 2003 to January 2009, 137 patients were recruited into cohort 1 (figure 1a). Two (1.4\%) patients were not assessed for RPVO because they were lost to follow-up; in six (4.4\%), the follow-up $V^{\prime} / Q^{\prime}$ lung scans were missing. Plasma samples for fibrin(ogen) laboratory analysis from $27(20.9 \%)$ subjects were unavailable. There were no significant differences between the 35 patients excluded from cohort 1 and the included patients from cohort 1 in age, sex, time from symptoms to diagnosis or residual obstruction. Among the 102 subjects included in the analysis, 29 (28.4\%) had RPVO $\geqslant 10 \%$ (table 1). The mean \pm SD time from pulmonary embolism diagnosis to $V^{\prime} / Q^{\prime}$ lung scan was $8.3 \pm 2.5$ months and degree of persistent perfusion defect was $8.1 \pm 11.7 \%$.
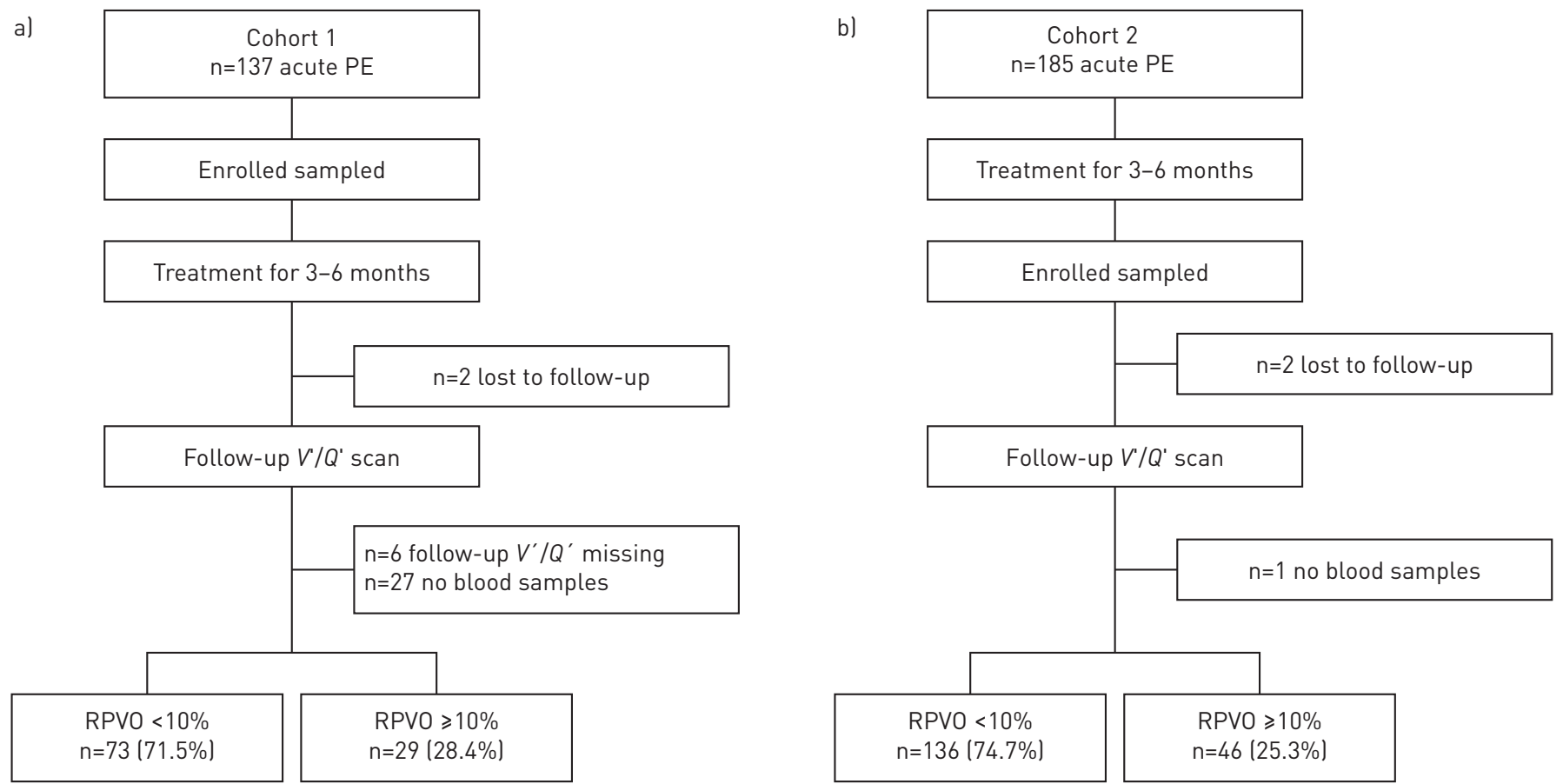

FIGURE 1 Study outline for both cohorts. a) In cohort 1, out of 137 subjects recruited the time of acute pulmonary embolism (PE) diagnosis, 102 could be evaluated, $29(28.4 \%)$ of whom had residual pulmonary vascular obstruction (RPVO) of $\geqslant 10 \%$; b) in cohort 2 , out of the 185 subjects recruited after long-term treatment for acute pulmonary embolism, 182 could be evaluated, 46 (25.3\%) of whom developed RPVO of $\geqslant 10 \%$. 
TABLE 1 Comparison of characteristics of cohort 1 and cohort 2

\begin{tabular}{|c|c|c|c|}
\hline & Cohort 1 & Cohort 2 & p-value $\#$ \\
\hline Subjects $\mathrm{n}$ & 102 & 182 & \\
\hline Age years & $52.2 \pm 19.6$ & $61.3 \pm 18.5$ & 0.0002 \\
\hline Male & 45.1 & 36.3 & 0.1644 \\
\hline VTE provoked & 52.9 & 54.4 & 0.9013 \\
\hline Recent travel for $>3 \mathrm{~h}$ & 12.7 & 12.1 & 0.8532 \\
\hline Trauma or LE fracture within 3 months & 10.8 & 9.3 & 0.6838 \\
\hline Bed rest & 8.8 & 20.3 & 0.0118 \\
\hline Surgery or anaesthesia within 3 months & 9.9 & 17.6 & 0.1153 \\
\hline Family history of VTE & 31.4 & 18.0 & 0.0121 \\
\hline Active cancer & 2.0 & 12.1 & 0.003 \\
\hline Concomitant DVT & 34.3 & 54.9 & 0.0009 \\
\hline Time from first symptom to diagnosis days & $11.2 \pm 17.1$ & $14.3 \pm 28.6$ & 0.0409 \\
\hline Diagnosis by CTPA, rather than $V^{\prime} / Q^{\prime}$ & 74.5 & 71.4 & 0.6778 \\
\hline Initial pulmonary vascular obstruction $\%$ & $38.8 \pm 25.9$ & $34.1 \pm 22.9$ & 0.1383 \\
\hline Thrombolysis & 0 & 5.5 & 0.0157 \\
\hline Inferior vena caval filter & 0 & 4.9 & 0.0288 \\
\hline Duration of anticoagulation days & $212.5 \pm 82.80$ & $231.8 \pm 171.42$ & 0.91 \\
\hline Extended-duration anticoagulation & 16.7 & 24.2 & 0.1752 \\
\hline Interval from diagnosis to study $V^{\prime} / Q^{\prime}$ scan days & $258 \pm 75$ & $330 \pm 111$ & $<0.0001$ \\
\hline Factor $\mathbf{V}_{\text {Leiden }}$ & 15.9 & 6.2 & 0.025 \\
\hline Prothrombin 20210a & 13.0 & 5.6 & 0.0637 \\
\hline Fibrinogen Aa T312A & 57.6 & 37.3 & 0.0019 \\
\hline Antithrombin" IU.dL $\mathrm{dL}^{-1}$ & $97.54 \pm 12.95$ & $96.50 \pm 11.13$ & 0.5982 \\
\hline Protein $\mathbf{C}^{+} \mathbf{I U} \cdot \mathbf{d L}^{-1}$ & $98.45 \pm 24.81$ & $104.10 \pm 19.69$ & 0.0749 \\
\hline Protein $\mathbf{S}^{\S} \mathbf{I U} \cdot \mathbf{d L}^{-1}$ & $89.18 \pm 20.76$ & $89.60 \pm 21.53$ & 0.9643 \\
\hline Fibrinogen $\mathbf{g} \cdot \mathbf{L}^{-1}$ & $4.28 \pm 1.13$ & $3.52 \pm 0.89$ & $<0.0001$ \\
\hline Platelet $\mathbf{G} \cdot \mathbf{L}^{-1}$ & $258.8 \pm 86.43$ & $258.9 \pm 68.48$ & 0.6496 \\
\hline Prothrombin ratio $\%$ control & $78.97 \pm 18.52$ & $83.75 \pm 20.38$ & 0.001 \\
\hline Thrombin \% control & $83.35 \pm 30.13$ & $93.76 \pm 27.03$ & 0.0811 \\
\hline Factor V \% control & $106.2 \pm 26.58$ & $103.8 \pm 25.32$ & 0.9201 \\
\hline Factor VII \% control & $86.67 \pm 40.75$ & $100.30 \pm 34.10$ & 0.1526 \\
\hline
\end{tabular}

Data are presented as mean \pm SD or $\%$, unless otherwise stated. Wilcoxon-Mann-Whitney testing was used to calculate $p$-values for continuous variables. Fisher's exact test was used for binary variables. VTE: venous thromboembolism; LE : lower extremity; DVT: deep vein thrombosis; CTPA: computed tomography pulmonary angiogram; $V^{\prime} / Q^{\prime}$ : ventilation/perfusion ratio. ${ }^{\#}$ : $p$-value for comparison between cohort 1 and cohort 2; ๆ: not measured in 23 out of 102 subjects in cohort 1 and 19 out of 182 subjects in cohort $2 ;^{+}$: protein $\mathrm{C}$ values were not measured in 38 out of 102 subjects in cohort 1 and 27 out of 182 subjects in cohort 2; ${ }^{\S}$ : protein $S$ values were not measured in 42 out of 102 subjects in cohort 1 and 35 out of 182 subjects in cohort 2 .

Between February 1999 and May 2006, 185 patients were recruited into cohort 2 (figure 1b). Two (1.0\%) patients were lost to follow-up. Plasma samples for fibrin(ogen) laboratory analysis were unavailable from one $(0.5 \%)$ subject. The excluded patients were not outliers on any of the studied characteristics. Among the remaining 182 subjects, $46(25.3 \%)$ had $\mathrm{RPVO} \geqslant 10 \%$. The mean \pm sD time from pulmonary embolism diagnosis to $V^{\prime} / Q^{\prime}$ lung scan was $10.9 \pm 3.7$ months and mean RPVO was $6.8 \pm 12.7 \%$.

The subject characteristics are compared between the two cohorts in table 1 (a comprehensive list is included in online supplementary appendix 2). Cohort 1 was significantly younger than cohort 2 $(52.2 \pm 19.6$ years versus $61.3 \pm 18.5$ years, $\mathrm{p}=0.0002)$. Cohort 1 had a lower prevalence of cancer $(2.0 \%$ versus $12.1 \%, \mathrm{p}=0.003)$, a lower prevalence of bed rest ( $8.8 \%$ versus $20.3 \%, \mathrm{p}=0.01$ ), a lower use of thrombolytics $(0 \%$ versus $5.5 \%, \mathrm{p}=0.02)$ and a lower use of inferior vena cava filters $(0 \%$ versus $4.5 \%, \mathrm{p}=0.03)$ than cohort 2. However, family histories of VTE were more common in cohort 1 than in cohort $2(31.4 \%$ versus $18.0 \%, \mathrm{p}=0.01)$. Pulmonary embolism was diagnosed somewhat earlier after the onset of symptoms in cohort 1 than in cohort $2(11.2 \pm 17.1$ days versus $14.3 \pm 28.6$ days, $\mathrm{p}=0.04)$.

A univariate analysis of the factors associated with RPVO is displayed in table 2. In both cohorts, the initial pulmonary vascular obstructions were smaller among those who recovered without RPVO, than in those with RPVO. Notably, a CTEPH-associated mutation (fibrinogen SanDiegoIII $_{\text {) }}$ [20] was found in one subject from cohort 1 , who had RPVO of $29.5 \%$. 
TABLE 2 Univariate comparison of characteristics between patients with residual pulmonary vascular obstruction (RPVO) and without

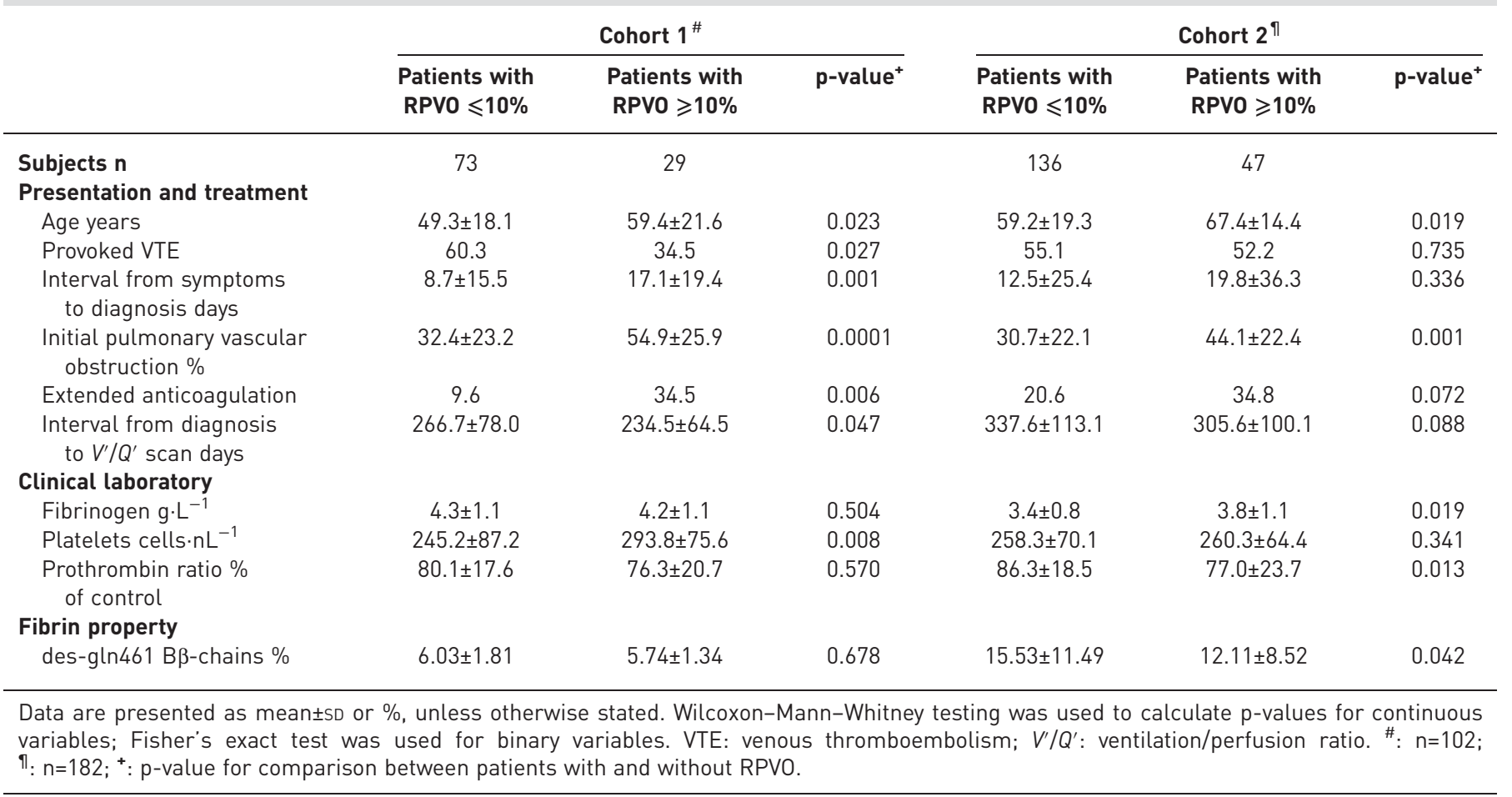

Derivation of RPVO predictive model in cohort 1

Prediction model creation and selection was performed on 94 subjects in cohort 1 who had complete clinical and fibrin-related datasets. When all candidate variables were considered, the optimal model to predict the probability of RPVO included the initial extent of vascular obstruction, fibrinogen B $\beta$-chain monosialylation, the days between symptoms and diagnosis and fibrinogen- $\gamma^{\prime}$ (table 3). The goodness of fit of the model was highly significant (difference between null and residual deviances $=21.8$, Chi-squared $\mathrm{p}=0.0002)$. The area under the ROC curve (AUC) was $0.762(\mathrm{p}=0.0001)$. The sensitivity was $0.679(95 \%$ CI $0.476-0.841)$ and the specificity was 0.727 (95\% CI $0.604-0.830)$.

\section{Validation of RPVO predictive model in cohort 2}

The optimal predictive model derived from all candidate variables in cohort 1 was applied to the 141 subjects in cohort 2 who had complete datasets. The AUC was $0.693(\mathrm{p}=0.0005)$. The sensitivity was 0.622 (95\% CI 0.448-0.775) and the specificity was 0.692 (95\% CI $0.595-0.777)$.

\begin{tabular}{|c|c|c|}
\hline Candidate predictive variables & OR $(95 \% \mathrm{CI})$ & p-value $\#$ \\
\hline \multicolumn{3}{|l|}{ Presentation and treatment } \\
\hline Initial obstruction & $1.041(1.018-1.064)$ & 0.000369 \\
\hline Interval symptoms to diagnosis & $1.026(0.997-1.056)$ & 0.078758 \\
\hline \multicolumn{3}{|l|}{ Fibrin property } \\
\hline $\mathrm{B} \beta$-chain monosialylation & 1.074 (0.986-1.168) & 0.100657 \\
\hline Fibrinogen- $\gamma^{\prime}$ & $0.641(0.419-0.980)$ & 0.040079 \\
\hline Model goodness of fit ${ }^{\text {I }}$ & \multicolumn{2}{|c|}{0.00022} \\
\hline
\end{tabular}

Candidate predictors with $\mathrm{p}$-values listed in the table were incorporated in the corresponding models that had been selected by the Akaike information criterion to be optimally predictive of RPVO after acute pulmonary embolism for the cohort. ": p-value from multivariate analysis; ": deviance-based goodness of fit, comparing the fit of each model with the fit of the null model. 
TABLE 4 Predictors of residual pulmonary vascular obstruction (RPVO) in cohort 1, considering only the initial obstruction and fibrin properties

\begin{tabular}{|c|c|c|}
\hline Candidate predictive variables & OR $(95 \% \mathrm{CI})$ & p-value ${ }^{\#}$ \\
\hline \multicolumn{3}{|l|}{ Presentation and treatment } \\
\hline Initial obstruction & $1.054(1.028-1.080)$ & 0.00003 \\
\hline \multicolumn{3}{|l|}{ Fibrin property } \\
\hline $\mathrm{B} \beta$-chain monosialylation & $1.095(0.994-1.206)$ & 0.0655 \\
\hline Fibrin clot lysis rate & $10.872(0.349-338)$ & 0.1737 \\
\hline Fibrinogen- $\gamma^{\prime}$ & $0.614(0.386-0.975)$ & 0.0388 \\
\hline Model goodness of fit ${ }^{\pi}$ & \multicolumn{2}{|c|}{0.00003} \\
\hline
\end{tabular}

Candidate predictors with $\mathrm{p}$-values listed in the table were incorporated in the corresponding models that had been selected by the Akaike information criterion to be the optimally predictive of RPVO after acute pulmonary embolism for the cohort. " : p-value from multivariate analysis; " : deviance-based goodness of fit, comparing the fit of each model with the fit of the null model.

\section{Derivation of RPVO predictive model in cohort 1 using fibrinogen properties}

When the candidate predictor pool excluded the clinical and demographic data, and encompassed only the initial extent of obstruction and fibrinogen data, the best prediction model's goodness of fit remained highly significant (difference between null and residual deviances $=26.2$, Chi-squared $\mathrm{p}=0.00003$ ). Initial obstruction and fibrinogen $\mathrm{B} \beta$-chain monosialylation remained as predictors of RPVO in that model, which also included fibrinogen- $\gamma^{\prime}$ and the fibrin lysis rate (table 4). The AUC was 0.802. However, when the candidate predictor pool also excluded the fibrinogen data, Chi-squared tests of residual deviances disclosed that the best-fit models did not predict RPVO as well as when fibrinogen data were included (difference between the two models' deviances=9.0, Chi-squared $\mathrm{p}=0.03$ ).

\section{Derivation of RPVO predictive model in cohort 2 using predictor pool from cohort 1}

A prediction model was created for cohort 2, with the pool of candidate predictors restricted to only the four predictors identified by the model creation and predictor selection process performed for cohort 1. When only those candidate variables were considered, the optimal model to predict the probability of RPVO included the initial extent of vascular obstruction, fibrinogen B $\beta$-chain monosialylation and the days between symptoms and diagnosis. Fibrinogen $\gamma^{\prime}$ was no longer included as a significant predictor. The goodness of fit of the model remained highly significant (difference between null and residual deviances $=18.8$, Chi-squared $\mathrm{p}=0.0009$ ). The AUC was 0.702 .

In a post hoc analysis, the median extent of B $\beta$-chain monosialylation among the entire (two-cohort) group was $59.2 \%$. Among the half of patients with B $\beta$-chain monosialylation below the median level, 19.4\% had RPVO, whereas $31.9 \%$ of those with higher levels of B $\beta$-chain monosialylation had RPVO $(\mathrm{p}=0.0169)$.

\section{Discussion}

We followed two cohorts of patients after acute pulmonary embolism to determine the factors that were most predictive of RPVO. High proportions of monosialylation of the fibrinogen B $\beta$-chain were consistently predictive of RPVO across the two cohorts. Among the other candidate variables, only the initial degree of vascular obstruction was more predictive of RPVO, which is in agreement with our previous observations [2].

We defined RPVO as persistent lung perfusion defects in $\geqslant 10 \%$ of the lungs (i.e. in at least two lung segments). Although we did not objectively measure quality of life or other symptomatic outcomes in the current study, we previously reported that our definition of RPVO, which occurred in $29 \%$ of acute pulmonary embolism patients, was significantly associated with dyspnoea, elevated pulmonary artery pressures and exercise intolerance [2]. Among pulmonary embolism patients with this degree of RPVO, $16 \%$ were subsequently diagnosed with CTEPH [2].

The predictors selected by the model were highly associated with the subsequent development of RPVO ( $p=0.0001$ in the derivation cohort and $p=0.0005$ in the validation cohort). The diagnostic accuracies of the model were not sufficiently high to exclude of predict RPVO for clinical purposes (AUC 0.762 and 0.693 in the derivation and validation cohorts, respectively). However, the association between RPVO and fibrinogen properties provides insights into the mechanism whereby some patients develop RPVO or even CTEPH after acute pulmonary embolism. No association with genetic thrombophilia has been identified [2], which 
raises the possibility that impairments in the process of fibrinolysis are responsible [33]. We focused our investigation on fibrin itself, since the resistance of purified fibrin clots to lysis has been observed in some patients with CTEPH $[19,20]$ and, to a lesser extent, in some pulmonary embolism patients [33, 34]. We used as our model the properties of fibrin [21, 22] that we had observed in CTEPH-associated dysfibrinogaenemias [20]. We reasoned that variations in post-translational modification patterns might influence fibrin formation and lysis $[35,36]$ less severely, but more commonly, than CTEPH-associated dysfibrinogaenemias and that they may have a role in persistence of thromboemboli after acute pulmonary embolism.

Our results support the hypothesis that attenuation of the body's normal mechanisms for resolution of fibrin-rich thrombi is an important pathophysiological step in the aetiology of chronic syndromes after venous thromboembolism $[5,18]$. One possible mechanism is that properties of the fibrin clot would delay or limit plasmin-mediated fibrinolysis or modify other clot resolution mechanisms such as angiogenic and inflammation responses [18]. Our observations suggest that structural attributes of fibrin may influence fibrin clot structure sufficiently to contribute to poor clot resolution and chronic disease after acute pulmonary embolism.

High proportions of monosialiated B $\beta$-chains were predictive of RPVO in both pulmonary embolism cohorts studied. Sialylation occurs during fibrinogen formation and has various effects on fibrin polymer organisation and resolution due to the electrostatic effect of the negatively charged sialic acid. Experimental sialic acid removal results in disordered fibrin clot formation $[35,37,38]$, while excessive sialylation of $\mathrm{B} \beta$-chains and $\gamma$-chains also results in abnormal clots $[22,36]$. Monosialylation is increased when clotting might be beneficial, such as during pregnancy [39] and acute infection [40].

The results for the two cohorts we studied differed somewhat in the predictive power of low fibrinogen- $\gamma^{\prime}$, which has been implicated in the regulation of fibrin cross-linking. The differences might be due to chance. Alternatively, they might reflect subtle differences between the two cohorts or even different implications of fibrin(ogen) characteristics at the time of the acute pulmonary embolism (when blood samples were collected from the first cohort) and several months later (when the second cohort was sampled).

Our study is limited by the fact that we did not independently confirm the clinical significance of RPVO in our patients by objective measurements of dyspnoea, haemodynamics or subsequent clinical outcomes after completion of the study protocol. However, we have validated the association between RPVO and those findings in a previous series of similar pulmonary embolism patients [2]. We also acknowledge that $20 \%$ of patients in the first cohort were excluded because they did not have adequate plasma samples for fibrinogen analysis, which could have introduced a potential for bias in the analysis of that cohort. While we did not disclose an association between the type of acute or long-term anticoagulation therapy and RPVO, the study was performed prior to the widespread use of direct oral anticoagulants, so we cannot comment on the influence of those therapies. Finally, despite the consistency of our findings in both cohorts who had similar rates of RPVO, they should be confirmed in subsequent prospective studies.

\section{Conclusion}

The extent of pulmonary vascular obstruction at the onset of pulmonary embolism, as well as fibrinogen B $\beta$-chain monosialylation, were predictive of RPVO after acute pulmonary embolism in the two cohorts studied. Our results confirm our previous observations and support the role of fibrin properties in the resolution or persistence of thrombi after acute pulmonary embolism, which is similar to the role played in the development of CTEPH. It is possible that abnormal fibrin properties could help identify acute pulmonary embolism patients at high risk for RPVO for whom adjuvant therapy to modify disease progression might be evaluated. For example, since fibrinogen properties such as fibrinogen B $\beta$-sialylation may be influenced pharmacologically [39], the effect on RPVO of such interventions to alter fibrinogen or the body's response to fibrin may have clinical implications.

Acknowledgements: The authors would like to thank Russell Doolittle (University of California San Diego, San Diego, CA, USA) for his helpful advice and suggestions on the experimental methods and manuscript. Jess Mandel (University of California San Diego, San Diego, CA, USA) also provided helpful comments on the manuscript.

Author contributions: B. Planquette recruited patients, performed experiments and helped write the manuscript; O. Sanchez, J. Emmerich and G. Meyer helped design the study and contributed to the manuscript; J.J. Marsh, P.G. Chiles, R.E. Moore and G.B. Gugiu performed laboratory experiments and contributed to the manuscript; G. Le Gal planned analysis and contributed to the manuscript; T. Wolfson and A.C. Gamst performed statistical analyses and contributed to the manuscript. T.A. Morris is the study primary investigator, planned the study, performed experiments and contributed to the manuscript. 
Conflict of interest: B. Planquette has nothing to disclose. O. Sanchez reports grants, personal fees and non-financial support from Bayer, grants, personal fees and non-financial support from MSD, grants and non-financial support from Actelion, personal fees and non-financial support from BMS-Pfizer, grants from Daiichi Sankyo, personal fees from Chiesi and non-financial support from Boehringer Ingelheim, outside the submitted work. J.J. Marsh has nothing to disclose. P.G. Chiles has nothing to disclose. J. Emmerich has nothing to disclose. G. Le Gal has acted as a co-investigator in clinical trials for Portola Pharmaceuticals, Boehringer Ingelheim, Pfizer, Bristol-Myers Squibb, LEO Pharma, Daiichi Sankyo and Bayer, and has received honoraria, not taken as salary from Bayer, Pfizer, LEO Pharma, Sanofi and bioMérieux, outside the submitted work. G. Meyer reports grants from Bayer and non-financial support from Leo Pharma, BMS-Pfizer and Daiichi Sankyo, outside the submitted work. T. Wolfson has nothing to disclose. A. C. Gamst has nothing to disclose. R.E. Moore has nothing to disclose. G.B. Gugiu has nothing to disclose. T.A. Morris reports grants from Bayer Pharmaceuticals, during the conduct of the study; personal fees for consulting from Bayer Pharmaceuticals, and personal fees for acting as an expert witness from Faegre Baker Daniels, outside the submitted work

Support statement: The study was funded by research grants from Bayer Pharmaceuticals, Fonds de Recherche en Santé Respiratoire, France, R01HL095089 and through collaboration with the UCSD DNA Sequencing Facility (supported, in part, by grant 2 P30 CA023100-23 from the NIH) for performing DNA sequence analysis; and the City of Hope Mass Spectrometry and Proteomics Core Facility (supported, in part, by grant CA033572 from the NIH) for performing fibrinogen mass spectrometry analysis. The authors had full discretion over the study design, performance and creation of the manuscript. Funding information for this article has been deposited with the Crossref Funder Registry.

\section{References}

1 Moser KM, Bloor CM. Pulmonary vascular lesions occurring in patients with chronic major vessel thromboembolic pulmonary hypertension. Chest 1993; 103: 685-692.

2 Sanchez O, Helley D, Couchon S, et al. Perfusion defects after pulmonary embolism: risk factors and clinical significance. J Thromb Haemost 2010; 8: 1248-1255.

3 Klok FA, van Kralingen KW, van Dijk AP, et al. Quality of life in long-term survivors of acute pulmonary embolism. Chest 2010; 138: 1432-1440.

4 Wartski M, Collignon MA. Incomplete recovery of lung perfusion after 3 months in patients with acute pulmonary embolism treated with antithrombotic agents. J Nucl Med 2000; 41: 1043-1048.

5 Klok FA, van der Hulle T, den Exter PL, et al. The post-PE syndrome: a new concept for chronic complications of pulmonary embolism. Blood Rev 2014; 28: 221-226.

6 Planquette B, Ferré A, Peron J, et al. Residual pulmonary vascular obstruction and recurrence after acute pulmonary embolism. A single center cohort study. Thromb Res 2016; 148: 70-75.

7 Meneveau N, Ider O, Seronde MF, et al. Long-term prognostic value of residual pulmonary vascular obstruction at discharge in patients with intermediate- to high-risk pulmonary embolism. Eur Heart J 2013; 34: 693-701.

8 Phear D. Pulmonary embolism. A study of late prognosis. Lancet 1960; 2: 832-835.

9 Prediletto R, Paoletti P, Fornai E, et al. Natural course of treated pulmonary embolism. Evaluation by perfusion lung scintigraphy, gas exchange, and chest roentgenogram. Chest 1990; 97: 554-561.

10 Paraskos JA, Adelstein SJ, Smith RE, et al. Late prognosis of acute pulmonary embolism. N Engl J Med 1973; 289: 55-58.

11 Donnamaria V, Palla A, Petruzzelli S, et al. Early and late follow-up of pulmonary embolism. Respiration 1993; 60: 15-20.

12 Wan $\mathrm{T}$, Rodger $\mathrm{M}$, Zeng W, et al. Residual pulmonary embolism as a predictor for recurrence after a first unprovoked episode: results from the REVERSE cohort study. Thromb Res 2018; 162: 104-109.

13 Pesavento R, Filippi L, Palla A, et al. Impact of residual pulmonary obstruction on the long-term outcome of patients with pulmonary embolism. Eur Respir J 2017; 49: 1601980.

14 Pengo V, Lensing AW, Prins $\mathrm{MH}$, et al. Incidence of chronic thromboembolic pulmonary hypertension after pulmonary embolism. N Engl J Med 2004; 350: 2257-2264.

15 Guérin L, Couturaud F, Parent F, et al. Prevalence of chronic thromboembolic pulmonary hypertension after acute pulmonary embolism. Prevalence of CTEPH after pulmonary embolism. Thromb Haemost 2014; 112: 598-605.

16 Piazza G, Goldhaber SZ. Chronic thromboembolic pulmonary hypertension. N Engl J Med 2011; 364: 351-360.

17 Lang IM, Madani M. Update on chronic thromboembolic pulmonary hypertension. Circulation 2014; 130: 508-518.

18 Fernandes T, Planquette B, Sanchez O, et al. From acute to chronic thromboembolic disease. Ann Am Thorac Soc 2016; 13: Suppl. 3, S207-S214.

19 Morris TA, Marsh JJ, Chiles PG, et al. Fibrin derived from patients with chronic thromboembolic pulmonary hypertension is resistant to lysis. Am J Respir Crit Care Med 2006; 173: 1270-1275.

20 Morris TA, Marsh JJ, Chiles PG, et al. High prevalence of dysfibrinogenemia among patients with chronic thromboembolic pulmonary hypertension. Blood 2009; 114: 1929-1936.

21 Marsh JJ, Guan HS, Li S, et al. Structural insights into fibrinogen dynamics using amide hydrogen/deuterium exchange mass spectrometry. Biochemistry 2013; 52: 5491-5502.

22 Marsh JJ, Chiles PG, Liang NC, et al. Chronic thromboembolic pulmonary hypertension-associated dysfibrinogenemias exhibit disorganized fibrin structure. Thromb Res 2013; 132: 729-734.

23 Miller RF, O’Doherty MJ. Pulmonary nuclear medicine. Eur J Nucl Med 1992; 19: 355-368.

24 Meyer G, Collignon MA, Guinet F, et al. Comparison of perfusion lung scanning and angiography in the estimation of vascular obstruction in acute pulmonary embolism. Eur J Nucl Med 1990; 17: 315-319.

25 PIOPED Investigators. Value of the ventilation/perfusion scan in acute pulmonary embolism. Results of the prospective investigation of pulmonary embolism diagnosis (PIOPED). JAMA 1990; 263: 2753-2759.

26 Qanadli SD, El Hajjam M, Vieillard-Baron A, et al. New CT index to quantify arterial obstruction in pulmonary embolism: comparison with angiographic index and echocardiography. AJR Am J Roentgenol 2001; 176: $1415-1420$. 
27 Qi J, Kreutzer DL. Fibrin activation of vascular endothelial cells. Induction of IL-8 expression. J Immunol 1995; 155: 867-876.

28 Martinez J, Ferber A, Bach TL, et al. Interaction of fibrin with VE-cadherin. Ann NY Acad Sci 2001; 936: 386-405.

29 Bunce LA, Sporn LA, Francis CW. Endothelial cell spreading on fibrin requires fibrinopeptide B cleavage and amino acid residues 15-42 of the beta chain. J Clin Invest 1992; 89: 842-850.

30 Burnham KP, Anderson DR. Information and likelihood theory: a basis for model selection and inference. In: Model Selection and Multimodel Inference: A Practical Information-Theoretic Approach. New York, Springer-Verlag, 2002; pp. 49-97.

31 Akaike H. Information theory and an extension of the maximum likelihood principle. In: Petrov BN, Csaki F, eds. International Symposium on Information Theory. Budapest, Akademia Kiado, 1973; pp. 267-281.

32 Hosmer DW, Lemeshow S. Assessing the Fit of the Model. Applied Logistic Regression. 2nd Edn. New York, John Wiley \& Sons, 2000; pp. 143-202.

33 Lami D, Cellai AP, Antonucci E, et al. Residual perfusion defects in patients with pulmonary embolism are related to impaired fibrinolytic capacity. Thromb Res $2014 ; 134: 737-741$.

34 Miniati M, Fiorillo C, Becatti M, et al. Fibrin resistance to lysis in patients with pulmonary hypertension other than thromboembolic. Am J Respir Crit Care Med 2010; 181: 992-996.

35 Okude M, Yamanaka A, Morimoto Y, et al. Sialic acid in fibrinogen: effects of sialic acid on fibrinogen-fibrin conversion by thrombin and properties of asialofibrin clot. Biol Pharm Bull 1993; 16: 448-452.

36 Morris TA, Marsh JJ, Chiles PG, et al. Abnormally sialylated fibrinogen $\gamma$-chains in a patient with chronic thromboembolic pulmonary hypertension. Thromb Res 2007; 119: 257-259.

37 Dang CV, Shin CK, Bell WR, et al. Fibrinogen sialic acid residues are low affinity calcium-binding sites that influence fibrin assembly. J Biol Chem 1989; 264: 15104-15108.

38 Martinez J, Palascak J, Peters C. Functional and metabolic properties of human asialofibrinogen. J Lab Clin Med 1977; 89: 367-377.

39 Maghzal GJ, Brennan SO, George PM. The sialic acid content of fibrinogen decreases during pregnancy and increases in response to fibrate therapy. Thromb Res 2005; 115: 293-299.

40 Brennan SO. Variation of fibrinogen oligosaccharide structure in the acute phase response: possible haemorrhagic implications. BBA Clin 2015; 3: 221-226. 